JouRnal OF Universal MATHEMATICS

Vol.1 No.2 PP.245-257 (2018)

ISSN-2618-5660

\title{
EXISTENCE OF POSITIVE SOLUTIONS OF NONLINEAR FRACTIONAL QUADRATIC DIFFERENTIAL EQUATIONS
}

\author{
K. HILAL, Y. ALLAOUI, AND K. GUIDA
}

\begin{abstract}
In this work, we prove the existence as well as approximations of the positive solutions for an initial value problem of nonlinear fractional quadratic differential equations. We use some properties of the Mittag-Leffler functions and its relationship with fractional calculus. Also we obtain some results regarding the existence of positive solutions using the Dhage iterative method embodied in a recent hybrid fixed point theorem of Dhage in partially ordered normed linear spaces.
\end{abstract}

\section{INTRODUCTION}

Fractional differential equations have received increasing attention during recent years due to their application in various fields of science and engineering, such as viscoelasticity, electrochemistry, porous media and electromagnetism [7],[9],[10],[11]. For more details on this theory and application, we refer the readers to I.Podlubny [14], Miller and Ross [13], Kilbas and all [8] and Zhou [16].

Very recently, the study of existence and approximation of the solutions for the hybrid differential equations is initiated in Dhage [3] and Dhage and all [1],[2] via hybrid fixed point theory.

C.Dhage and B.Dhage [3] discussed the following quadratic differential equations.

$$
\begin{aligned}
\frac{d}{d t}\left[\frac{x(t)}{f(t, x(t))}\right]+\lambda\left[\frac{x(t)}{f(t, x(t))}\right] & =g(t, x(t)), t \in J=\left[t_{0}, t_{0}+a\right] \\
x\left(t_{0}\right) & =x_{0} \in \mathbb{R},
\end{aligned}
$$

for $\lambda \in \mathbb{R}^{+}$where $f: J \times \mathbb{R} \longrightarrow \mathbb{R}^{*}$ and $g: J \times \mathbb{R} \longrightarrow \mathbb{R}$ are a continuous functions. They established the existence of positive solutions using the Dhage iterative method.

From this work, we develop the theory of fractional quadratic differential equations involving Caputo differential operators of order $0<\alpha<1$. We prove the existence of positive solutions of the following fractional quadratic differential equations (for

Date: July 1, 2018, accepted.

2000 Mathematics Subject Classification. Primary 34A08; Secondary 34A38.

Key words and phrases. Fractional differential equation, hybrid systems, Dhage iterative method, Approximate positive solution. 
short FQDE):

$$
\begin{aligned}
D^{\alpha}\left[\frac{x(t)}{f(t, x(t))}\right]-\lambda\left[\frac{x(t)}{f(t, x(t))}\right] & =g(t, x(t)), t \in J=[0,1] \\
x(0) & =x_{0} \in \mathbb{R},
\end{aligned}
$$

where $D^{\alpha}$ denotes the Caputo fractional derivative of order $\alpha$, where $0<\alpha<1$, $\lambda>0, f: J \times \mathbb{R} \longrightarrow \mathbb{R}^{*}$ is a continuous function and $g: J \times \mathbb{R} \longrightarrow \mathbb{R}$ is a $L^{1}-$ Caratheodory function.

We note that the (FQDE) (1) - (2) with $\lambda=0$ is considered by Hilal and Kajouni [5], [6].

The rest of this paper is organized as follows: In section 2, we give some preliminaries which are used in the sequal. In section 3 we prove our main results.

\section{Preliminaries}

In this section, we introduce some definitions and results which are used throughout this paper.

Definition 2.1. [15] Let $x \in C^{n}[0, \infty)$ and $n-1<\alpha<n$, where $n \in \mathbb{N}^{*}$, the Caputo's derivative of order $\alpha$ for function $x:[0, \infty) \longrightarrow \mathbb{R}$ can be written as

$$
D^{\alpha} x(t)=\frac{1}{\Gamma(n-\alpha)} \int_{0}^{t}(t-s)^{n-\alpha-1} x^{(n)}(s) d s .
$$

Definition 2.2. [15] The fractional integral of order $\alpha$ is defined as

$$
I^{\alpha} x(t)=\frac{1}{\Gamma(\alpha)} \int_{0}^{t}(t-s)^{\alpha-1} x(s) d s,
$$

which is called the Riemann-Liouville integral.

We give a useful lemma which plays an important role in the fractional calculus.

Definition 2.3. $[12,4]$ Let $\alpha, \beta>0$. The two-parameters Mittag-Leffler function $E_{\alpha, \beta}(t)$ is defined by the series expansion

$$
E_{\alpha, \beta}(t)=\sum_{i=0}^{\infty} \frac{t^{i}}{\Gamma(\alpha i+\beta)} .
$$

Especially, if $\beta=1, E_{\alpha, 1}(t)$ becomes the one-parameter Mittag-Leffler function $E_{\alpha}(t)$, i.e., $E_{\alpha, 1}(t)=E_{\alpha}(t)$.

Lemma 2.4. [12]

Let $0<\alpha<1$, and $\lambda \in \mathbb{R}$. Then for any $t \in[0, T]$, we have

(1)- Let $0<\alpha<1$, and $K, U \in \mathbb{R}^{n \times n}$. Then for any $t \in[0, T]$, it has

$\int_{0}^{t}(t-\tau)^{\alpha-1} E_{\alpha, \alpha}\left(-K(t-\tau)^{\alpha}\right) U\left(D^{\alpha} x\right)(\tau) d \tau=U x(t)-E_{\alpha}\left(\lambda^{\alpha}\right) U x(0)-K \int_{0}^{t}(t-s)^{\alpha-1} E_{\alpha, \alpha}\left(-K(t-s)^{\alpha}\right) U x(s) d s$ (2)-

$$
D_{0}^{\alpha}\left[\int_{0}^{t} f(t-s) g(s) d s\right](t)=\int_{0}^{t} D_{0}^{\alpha}[f(t)](s) g(t-s) d s+g(t) \lim _{t \rightarrow 0^{+}}\left[{ }_{t} I_{0^{+}}^{1-\alpha} f\right](t) .
$$


Lemma 2.5. $[8,4]$

(a)- $D_{a^{+}}^{\alpha} E_{\alpha}\left[\lambda(t-a)^{\alpha}\right](x)=\lambda E_{\alpha}\left[\lambda(x-a)^{\alpha}\right], \quad(\operatorname{Re}(\alpha)>0, \lambda \in \mathbb{C})$

(b)- $\left(I_{a+}^{\alpha^{\prime}}(t-a)^{\beta-1} E_{\mu, \beta}\left[\lambda(t-a)^{\mu}\right]\right)(x)=(x-a)^{\alpha^{\prime}+\beta-1} E_{\mu, \alpha^{\prime}+\beta}\left[\lambda(x-a)^{\mu}\right]$, with $\alpha^{\prime}>0, \beta>0$ and $\mu>0$.

(c)- $\int_{0}^{z} t^{\beta-1} E_{\alpha, \beta}\left(\lambda t^{\alpha}\right) d t=z^{\beta} E_{\alpha, \beta+1}\left(\lambda z^{\alpha}\right)$.

(d) $\left|E_{\alpha, \beta}(z)\right| \leq C_{1} \exp \left(\sigma|z|^{\rho}\right)$, for all $\sigma>1$ and $\rho=\frac{1}{\operatorname{Re}(\alpha)}$.

The following definitions are useful in the sequel.

We conserve the same definitions given in Dhage [3].

Let $\mathrm{E}$ denotes a partially ordered real-normed linear space with an order relation $\leq$ and the norm $\|\cdot\|$.

Definition 2.6. [3] A mapping $\mathcal{T}: E \longrightarrow E$ is called nondecreasing if it preserves the order relation $\leq$, that is if $x \leq y$ implies $\mathcal{T} x \leq \mathcal{T} y$ for all $x, y \in E$.

Definition 2.7. [3] A mapping $\mathcal{T}: E \longrightarrow E$ is called partially continuous at a point $a \in E$ if for $\varepsilon>0$ there exists a $\delta>0$ such that $\|\mathcal{T} x-\mathcal{T} a\|<\varepsilon$ whenever $x$ is comparable to $a$ and $\|x-a\|<\delta . \mathcal{T}$ called partially continuous on $E$ if it is partially continuous at every point of it.It is clear that if $\mathcal{T}$ is partially continuous on $E$, then it is continuous on every chain $C$ contained in $E$.

Definition 2.8. [3] A mapping $\mathcal{T}: E \longrightarrow E$ is called partially bounded if $\mathcal{T}(C)$ is bounded for every chain $C$ in $E$. $\mathcal{T}$ is uniformly partially bounded if all chain $\mathcal{T}(C)$ in $E$ are bounded by a unique constant.

$\mathcal{T}$ is called bounded if $\mathcal{T}(E)$ is a bounded subset of $E$.

Definition 2.9. [3] A mapping $\mathcal{T}: E \longrightarrow E$ is called partially compact if $\mathcal{T}(C)$ is a relatively compact subset of $E$ for all totally ordered sets or chain $C$ in $E$. $\mathcal{T}$ is uniformly partially compact if $\mathcal{T}(C)$ is a uniformly partially bounded and partially compact on $E . \mathcal{T}$ is called partially totally bounded if for any totally ordered and bounded subset $C$ of $E, \mathcal{T}(E)$ is a relatively compact subset of $E$. If $\mathcal{T}$ is partially continuous and partially totally bounded, then it is called partially completely continuous on $E$.

Definition 2.10. [3] An upper semi-continuous and nondecreasing function $\psi$ : $\mathbb{R}^{+} \longrightarrow \mathbb{R}^{+}$is called a $\mathcal{D}$ - function, provided $\psi(0)=0$. Let $(E, \leq,\|\|$.$) be a$ partially ordered normed linear space. A mapping $\mathcal{T}: E \longrightarrow E$ is called partially nonlinear $\mathcal{D}$ - Lipschitz if there exists a $\mathcal{D}$ - function $\psi: \mathbb{R}^{+} \longrightarrow \mathbb{R}^{+}$such that

$$
\|\mathcal{T} x-\mathcal{T} y\| \leq \psi(\|x-y\|),
$$

for all comparable elements $x, y \in E$. If $\psi(r)=k r, k>0$, then $\mathcal{T}$ is called a partially Lipschitz with a Lipschitz constant $k$.

Let $(E, \leq,\|\cdot\|)$ be a partially ordered normed linear algebra. We denote

$$
E^{+}=\{x \in E \mid x \geq \theta, \text { where } \theta \text { is the zero element of } E\}
$$




$$
\mathcal{K}=\left\{E^{+} \subset E \mid u v \in E^{+} \text {for all } u, v \in E^{+}\right\},
$$

and $\mathcal{P}_{c h}(E)$ is the set of all subsets of E.

The elements of the set $\mathcal{K}$ are called the positive vectors in $E$. The following lemma follows immediately from the definition of the set $\mathcal{K}$, which is oftentimes used in the hybrid fixed point theory of Banach algebras and applications to nonlinear differential and integral equations.

Lemma 2.11. [3] If $u_{1}, u_{2}, v_{1}, v_{2} \in \mathcal{K}$ are such that $u_{1} \leq v_{1}$ and $u_{2} \leq v_{2}$ then $u_{1} u_{2} \leq v_{2} v_{2}$.

Definition 2.12. [3]

An operator $T: E \rightarrow E$ is said to be positive if the range $R(T)$ of $T$ is such that $R(T) \subseteq \mathcal{K}$.

Theorem 2.13. [3] Let $(E, \leq,\|\cdot\|)$ be a regular partially ordered complete normed linear algebra such that the order relation $\leq$ and the norm $\|$.$\| in E$ are compatible in every compact chain of $E$. Let $\mathcal{A}, \mathcal{B} \longrightarrow \mathcal{K}$ be two nondecreasing operators such that

(a) $\mathcal{A}$ is partially bounded and partially nonlinear $\mathcal{D}-$ Lipschitz with $\mathcal{D}-$ function $\psi_{\mathcal{A}}$,

(b) $\mathcal{B}$ is partially continuous and uniformly partially compact,

(c) $M \psi_{\mathcal{A}}(r)<r, r>0$, where $M=\sup \left\{\|\mathcal{B}(C)\|: C \in \mathcal{P}_{c h}(E)\right\}$, and

(d) there exists an element $x_{0} \in X$ such that $x_{0} \leq \mathcal{A} x_{0} \mathcal{B} x_{0}$ or $x_{0} \geq \mathcal{A} x_{0} \mathcal{B} x_{0}$ then the operator equation

$$
\mathcal{A} x \mathcal{B} x=x
$$

has a positive solution $x^{*}$ in $E$ and the sequence $\left\{x_{n}\right\}$ of successive iterations defined by $x_{n+1}=\mathcal{A} x_{n} \mathcal{B} x_{n}, n=0,1, \ldots ;$ converges monotonically to $x^{*}$.

Let $\mathcal{C}(J \times \mathbb{R}, \mathbb{R})$ be the class of functions $g: J \times \mathbb{R} \longrightarrow \mathbb{R}$ such that

(i) the map $t \longrightarrow g(t, x)$ is measurable for each $x \in \mathbb{R}$, and

(ii) the map $x \longrightarrow g(t, x)$ is continuous for each $t \in J$.

The class $\mathcal{C}(J \times \mathbb{R}, \mathbb{R})$ is called the Caratheodory class of functions on $J \times \mathbb{R}$ which are Lebesgue integrable when bounded by a Lebesgue integrable function on J.

$L^{1}(J, \mathbb{R})$ denotes the space of Lebesgue integrable real-valued functions on $\mathrm{J}$ equipped with the norm $\|\cdot\|_{L^{1}}$ defined by

$$
\|x\|_{L^{1}}=\int_{0}^{1}|x(s)| d s .
$$

\section{MAin RESUlts:}

Definition 3.1. By a solution of the FQDE, we mean a function $x \in C^{1}(J, \mathbb{R})$ that satisfies 
(i) $t \longrightarrow \frac{x}{f(t, x)}$ is a continuously differentiable function for each $x \in \mathbb{R}$ and

(ii) $x$ satisfies the equations (1), (2) on $J$.

We consider the FQDE in the space $C(J, \mathbb{R})$ of continuous real-valued functions defined on $J$. We define a norm $\|$. $\|$ and the order relation $\leq$ in $C(J, \mathbb{R})$ by

$$
\|x\|=\sup _{t \in J}|x(t)|
$$

and

$$
x \leq y \Leftrightarrow x(t) \leq y(t) \text { for all } t \in J .
$$

Clearly, $C(J, \mathbb{R})$ is a Banach algebra with respect to above supremum norm and is also partially ordered with respect to the above partially order relation $\leq$. It is known that the partially ordered Banach algebra $C(J, \mathbb{R})$ has some nice properties with respect to the above order relation in it. The following lemma follows by an application of Arzela-Ascoli theorem.

Lemma 3.2. [3] Let $(C(J, \mathbb{R}), \leq, \|$. $\|)$ be a partially ordered Banach space with the norm $\|$.$\| and the order relation \leq$ defined by (3.1)-(3.2), respectively. Then, $\|$.$\| and \leq$ are compatible in every partially compact subset of $C(J, \mathbb{R})$.

Definition 3.3. A function $u \in C^{1}(J, \mathbb{R})$ is said to be a lower solution of the FQDE if the function $t \longrightarrow \frac{u(t)}{f(t, u(t))}$ is continuously differentiable and satisfies

$$
\begin{gathered}
D^{\alpha}\left[\frac{u(t)}{f(t, u(t))}\right]-\lambda\left[\frac{u(t)}{f(t, u(t))}\right] \leq g(t, u(t)), t \in J \\
u(0) \leq x_{0} .
\end{gathered}
$$

Similarly, a function $v \in C^{1}(J, \mathbb{R})$ is said to be upper solution of FQDE if satisfies the above property and inequalities with reverse sign.

Consider the following assumptions:

$\left(A_{0}\right)$ The map $x \longrightarrow \frac{x}{f(t, x)}$ is increasing in $\mathbb{R}$ for each $t \in J$.

$\left(A_{1}\right) f$ defines a function $f: J \times \mathbb{R} \longrightarrow \mathbb{R}^{+}$.

$\left(A_{2}\right)$ There exists a constant $M_{f}>0$ such that $0<f(t, x)<M_{f}$ for all $t \in J$ and $x \in \mathbb{R}$

$\left(A_{3}\right)$ There exists a $\mathcal{D}-$ function $\phi$, such that

$$
0 \leq f(t, x)-f(t, y) \leq \phi(x-y),
$$

for all $t \in J$ and $x, y \in \mathbb{R}, x \geq y$.

$\left(B_{1}\right) g$ defines a function $g: J \times \mathbb{R} \longrightarrow \mathbb{R}^{+}$.

$\left(B_{2}\right)$ There exists a function $h \in L^{1}(J, \mathbb{R})$ such that $g(t, x) \leq h(t)$ for all $t \in J$ and $x \in \mathbb{R}$.

$\left(B_{3}\right) g(t, x)$ is nondeceasing in $x$ for all $t \in J$.

$\left(B_{4}\right)$ The FQDE has a lower solution $u \in C^{1}(J, \mathbb{R})$.

Lemma 3.4. Suppose that hypothesis $A_{0}$ hold. Then a function $x \in C^{1}(J, \mathbb{R})$ is a solution of the FQDE 


$$
\begin{cases}D^{\alpha}\left[\frac{x(t)}{f(t, x(t))}\right]-\lambda\left[\frac{x(t)}{f(t, x(t))}\right] & =g(t) \\ x(0) & =x_{0} \in \mathbb{R},\end{cases}
$$

if and only if it is a solution of the nonlinear integral equation

$$
x(t)=f(t, x(t))\left(\frac{x_{0}}{f\left(0, x_{0}\right)} E_{\alpha}\left(\lambda t^{\alpha}\right)+\int_{0}^{t}(t-s)^{\alpha-1} E_{\alpha, \alpha}\left(\lambda(t-s)^{\alpha}\right) g(s) d s\right),
$$

for all $t \in J$.

Proof. Consider the problem $D^{\alpha}\left[\frac{x(t)}{f(t, x(t))}\right]-\lambda\left[\frac{x(t)}{f(t, x(t))}\right]=g(t)$.

By the relations (4.1.65) and (4.1.66) in [8], the integral equation has the solution

$$
\frac{x(t)}{f(t, x(t))}=\int_{0}^{t}(t-s)^{\alpha-1} E_{\alpha, \alpha}\left(\lambda(t-s)^{\alpha}\right) g(s) d s+\frac{x(0)}{f(0, x(0))} E_{\alpha}\left(\lambda t^{\alpha}\right) .
$$

According to the condition (1.2), we get

$$
x(t)=f(t, x(t))\left(\frac{x_{0}}{f\left(0, x_{0}\right)} E_{\alpha}\left(\lambda t^{\alpha}\right)+\int_{0}^{t}(t-s)^{\alpha-1} E_{\alpha, \alpha}\left(\lambda(t-s)^{\alpha}\right) g(s) d s\right) .
$$

Conversely, if we have

$$
x(t)=f(t, x(t))\left(\frac{x_{0}}{f\left(0, x_{0}\right)} E_{\alpha}\left(\lambda t^{\alpha}\right)+\int_{0}^{t}(t-s)^{\alpha-1} E_{\alpha, \alpha}\left(\lambda(t-s)^{\alpha}\right) g(s) d s\right),
$$

then

$$
D^{\alpha}\left(\frac{x(t)}{f(t, x(t))}\right)=D^{\alpha}\left(\int_{0}^{t}(t-s)^{\alpha-1} E_{\alpha, \alpha}\left(\lambda(t-s)^{\alpha}\right) g(s) d s\right)+D^{\alpha}\left(\frac{x_{0}}{f\left(0, x_{0}\right)} E_{\alpha}\left(\lambda t^{\alpha}\right)\right) .
$$

Substituting $x=t$ and $a=0$ in the relation ( $a$ ) in lemma 2.2, we have

$D^{\alpha} E_{\alpha}\left(\lambda t^{\alpha}\right)=\lambda E_{\alpha}\left(\lambda t^{\alpha}\right)$.

This implies that:

$D^{\alpha}\left(\frac{x_{0}}{f\left(0, x_{0}\right)} E_{\alpha}\left(\lambda t^{\alpha}\right)\right)=\frac{x_{0}}{f\left(0, x_{0}\right)} \lambda E_{\alpha}\left(\lambda t^{\alpha}\right)$.

Setting $F(u):=u^{\alpha-1} E_{\alpha, \alpha}\left(\lambda u^{\alpha}\right)$.

According to the relation (2) in lemma 2.1, we have 


$$
\begin{aligned}
& \left.D^{\alpha}\left[\int_{0}^{t} F(t-s) g(s) d s\right]=\int_{0}^{t} D^{\alpha}\left[s^{\alpha-1} E_{\alpha, \alpha}\left(\lambda s^{\alpha}\right)\right] g(t-s) d s\right]+g(t) \lim _{t \rightarrow 0^{+}} I^{1-\alpha} F(t) \\
& \left.=\int_{0}^{t} \lambda s^{\alpha-1} E_{\alpha, \alpha}\left(\lambda s^{\alpha}\right) g(t-s) d s\right]+g(t) \lim _{t \rightarrow 0^{+}} I^{1-\alpha} F(t) .
\end{aligned}
$$

Setting $t-s=u$, then we have

$$
D^{\alpha}\left[\int_{0}^{t} F(t-s) g(s) d s\right](t)=\int_{0}^{t} \lambda(t-u)^{\alpha-1} E_{\alpha, \alpha}\left(\lambda(t-u)^{\alpha}\right) g(u) d u+g(t) \lim _{t \rightarrow 0^{+}} I^{1-\alpha} F(t) .
$$

In the other hand, For $\alpha^{\prime}=1-\alpha, \mu=\beta=\alpha, a=0$ and $t=x$, in the relation (b), lemma 2.2, we have

$I^{1-\alpha} t^{\alpha-1} E_{\alpha, \alpha}\left[\lambda t^{\alpha}\right]=E_{\alpha, 1}\left[\lambda t^{\alpha}\right]$, wish implies

$g(t) \lim _{t \rightarrow 0^{+}} I^{1-\alpha} F(t)=g(t) \lim _{t \rightarrow 0^{+}} E_{\alpha, 1}\left[\lambda t^{\alpha}\right]=g(t)$.

In conclusion:

$$
\begin{aligned}
& D^{\alpha}\left(\frac{x(t)}{f(t, x(t))}\right)=\frac{x_{0}}{f\left(0, x_{0}\right)} \lambda E_{\alpha}\left(\lambda t^{\alpha}\right)+\int_{0}^{t} \lambda(t-u)^{\alpha-1} E_{\alpha, \alpha}\left(\lambda(t-s)^{\alpha}\right) g(s) d s+g(t) \\
& D^{\alpha}\left(\frac{x(t)}{f(t, x(t))}\right)-\lambda\left(\frac{x(t)}{f(t, x(t))}\right)=g(t) .
\end{aligned}
$$

Finally, for $t=0$ in the relation (3.3) and by the hypothesis $\left(A_{0}\right)$ which gives us $x(0)=x_{0}$ we get

$$
\frac{x(0)}{f(0, x(0))}=\frac{x_{0}}{f\left(0, x_{0}\right)}
$$

This completes the proof.

Theorem 3.5. Assume that hypothesis $\left(A_{0}\right)-\left(A_{3}\right)$ and $\left(B_{1}\right)-\left(B_{4}\right)$ hold. Furthermore, assume that

$$
\left(C_{1} \exp \left(2 \lambda^{\rho}\right)\left(\|h\|_{L^{1}}+\left|\frac{x_{0}}{f\left(0, x_{0}\right)}\right|\right)\right) \phi(r)<r, r>0,
$$

then, the FQDE has a positive solution $x^{*}$ defined on $J$ and the sequence $\left\{x_{n}\right\}_{n=1}^{\infty}$ of successive approximations defined by

$x_{n+1}(t)=\left[f\left(t, x_{n}(t)\right]\left(\frac{x_{0}}{f\left(0, x_{0}\right)} E_{\alpha}\left(\lambda t^{\alpha}\right)+\int_{0}^{t}(t-s)^{\alpha-1} E_{\alpha, \alpha}\left(\lambda(t-s)^{\alpha}\right) g\left(s, x_{n}(s)\right) d s\right)\right.$,

for $t \in \mathbb{R}$, where $x_{1}=u$, converges monotonically to $x^{*}$. 


\section{Proof}

Set $E=C(J, \mathbb{R})$, then, by lemma 3.1, every compact chain in $E$ possesses the compatibility property with respect to the norm $\|$. $\|$ and the order relation $\leq$ in $E$. By an application of lemma 3.2, the FQDE $(1),(2)$ is equivalent to the nonlinear integral equation,

$$
x(t)=\left[f(t, x(t)]\left(\frac{x_{0}}{f\left(0, x_{0}\right)} E_{\alpha}\left(\lambda t^{\alpha}\right)+\int_{0}^{t}(t-s)^{\alpha-1} E_{\alpha, \alpha}\left(\lambda(t-s)^{\alpha}\right) g(s, x(s)) d s\right) .\right.
$$

Define two operators $\mathcal{A}$ and $\mathcal{B}$ on $E$ by

$$
(\mathcal{A} x)(t)=f(t, x(t)), t \in J
$$

and

$$
(\mathcal{B} x)(t)=\frac{x_{0}}{f\left(0, x_{0}\right)} E_{\alpha}\left(\lambda t^{\alpha}\right)+\int_{0}^{t}(t-s)^{\alpha-1} E_{\alpha, \alpha}\left(\lambda(t-s)^{\alpha}\right) g(s, x(s)) d s .
$$

We shall show that the operators $\mathcal{A}$ and $\mathcal{B}$ satisfy all the conditions of the theorem 2.1. This is achieved in the series of following steps.

Step $I: \mathcal{A}$ and $\mathcal{B}$ are nondecreasing on $E$.

Let $x, y \in E$ be such that $x \geq y$. Then by hypothesis $\left(A_{3}\right)$, we obtain

$$
(\mathcal{A} x)(t)=f(t, x(t)) \geq f(t, y(t))=(\mathcal{A} y)(t)
$$

for all $t \in J$. This shows that $\mathcal{A}$ is nondcreasing operator on $E$ into $E$. Similarly using hypothesis $\left(B_{3}\right)$, it is shown that the operator $\mathcal{B}$ is also nondcreasing on $\mathrm{E}$ into itself. Thus, $\mathcal{A}$ and $\mathcal{B}$ are nondcreasing positive operators on $E$ into itself.

Step II: $\mathcal{A}$ is partially bounded and partially $\mathcal{D}$-Lipschitz on $E$.

Let $x \in E$ be arbitrary. Then by $\left(A_{2}\right)$,

$$
|(\mathcal{A} x)(t)|=f(t, x(t)) \leq M_{f}, \text { for all } t \in J .
$$

Taking supremum over t, we obtain $\|\mathcal{A} x\| \leq M_{f}$ and so, $\mathcal{A}$ is bounded. This further implies that $A$ is partially bounded on $E$.

Let $x, y \in E$ be such that $x \geq y$. Then,

$$
|(A x)(t)-(A y)(t)|=f(t, x(t))-f(t, y(t)) \leq \phi(|x(t)-y(t)|) \leq \phi(\|x-y\|)
$$

for all $t \in J$. Taking supremum over $t$, we obtain $\|\mathcal{A} x-\mathcal{A} y\| \leq \phi(\|x-y\|)$ for all $x, y \in E, x \geq y$. 
Hence, $\mathcal{A}$ is partially nonlinear $\mathcal{D}$-lipschitz on $E$ which further implies that $\mathcal{A}$ is partially continuous on $E$.

Step III: $\mathcal{B}$ is partially continuous on $E$.

Let $\left\{x_{n}\right\}_{n \in \mathbb{N}}$ be a sequence in a chain $C$ of $E$ such that $x_{n} \longrightarrow x$. Then, by the Lebesgue dominated convergence theorem, for all $t \in J$

$$
\begin{aligned}
\lim _{n \rightarrow \infty}\left(\mathcal{B} x_{n}\right)(t) & =\lim _{n \rightarrow \infty} \int_{0}^{t}(t-s)^{\alpha-1} E_{\alpha, \alpha}\left(\lambda(t-s)^{\alpha}\right) g\left(s, x_{n}(s)\right) d s+\lim _{n \rightarrow \infty} \frac{x_{0}}{f\left(0, x_{0}\right)} E_{\alpha}\left(\lambda t^{\alpha}\right) \\
& =\int_{0}^{t}(t-s)^{\alpha-1} E_{\alpha, \alpha}\left(\lambda(t-s)^{\alpha}\right) \lim _{n \rightarrow \infty} g\left(s, x_{n}(s)\right) d s+\frac{x_{0}}{f\left(0, x_{0}\right)} E_{\alpha}\left(\lambda t^{\alpha}\right) \\
& =\int_{0}^{t}(t-s)^{\alpha-1} E_{\alpha, \alpha}\left(\lambda(t-s)^{\alpha}\right) g(s, x(s)) d s+\frac{x_{0}}{f\left(0, x_{0}\right)} E_{\alpha}\left(\lambda t^{\alpha}\right) \\
& =(\mathcal{B} x)(t) .
\end{aligned}
$$

This shows that $\mathcal{B} x_{n}$ converges monotonically to $\mathcal{B} x$ pointwise on $J$.

Next, we will prove that $\left\{\mathcal{B} x_{n}\right\}$ is an equicontinuous sequence of functions in $E$.

Let $t_{1}, t_{2} \in J$ with $t_{1}<t_{2}$.

Setting $\psi(t)=\int_{0}^{t} h(s) d s$. Then

$$
\begin{aligned}
\left|\left(\mathcal{B} x_{n}\right)\left(t_{2}\right)-\left(\mathcal{B} x_{n}\right)\left(t_{1}\right)\right| \leq & \left|\int_{0}^{t_{2}}\left(t_{2}-s\right)^{\alpha-1} E_{\alpha, \alpha}\left(\lambda\left(t_{2}-s\right)^{\alpha}\right) g\left(s, x_{n}(s)\right) d s-\int_{0}^{t_{1}}\left(t_{1}-s\right)^{\alpha-1} E_{\alpha, \alpha}\left(\lambda\left(t_{1}-s\right)^{\alpha}\right) g\left(s, x_{n}(s)\right) d s\right| \\
& +\left|E_{\alpha}\left(\lambda t_{2}^{\alpha}\right)-E_{\alpha}\left(\lambda t_{1}^{\alpha}\right)\right|\left|\frac{x_{0}}{f\left(0, x_{0}\right)}\right| \\
\leq & \left|\int_{0}^{t_{1}}\left[\left(t_{2}-s\right)^{\alpha-1} E_{\alpha, \alpha}\left(\lambda\left(t_{2}-s\right)^{\alpha}\right)-\left(t_{1}-s\right)^{\alpha-1} E_{\alpha, \alpha}\left(\lambda\left(t_{1}-s\right)^{\alpha}\right)\right] g\left(s, x_{n}(s)\right) d s\right| \\
& +\left|\int_{t_{1}}^{t_{2}}\left(t_{2}-s\right)^{\alpha-1} E_{\alpha, \alpha}\left(\lambda\left(t_{2}-s\right)^{\alpha}\right) g\left(s, x_{n}(s)\right) d s\right|+\left|E_{\alpha}\left(\lambda t_{2}^{\alpha}\right)-E_{\alpha}\left(\lambda t_{1}^{\alpha}\right)\right|\left|\frac{x_{0}}{f\left(0, x_{0}\right)}\right| \\
\leq & \left|\int_{0}^{t_{1}}\right|\left(t_{2}-s\right)^{\alpha-1} E_{\alpha, \alpha}\left(\lambda\left(t_{2}-s\right)^{\alpha}\right)-\left(t_{1}-s\right)^{\alpha-1} E_{\alpha, \alpha}\left(\lambda\left(t_{1}-s\right)^{\alpha}\right)|| g\left(s, x_{n}(s)\right)|d s| \\
& +\left|\int_{t_{1}}^{t_{2}}\left(t_{2}-s\right)^{\alpha-1} E_{\alpha, \alpha}\left(\lambda\left(t_{2}-s\right)^{\alpha}\right)\right| g\left(s, x_{n}(s)\right)|d s|+\left|E_{\alpha}\left(\lambda t_{2}^{\alpha}\right)-E_{\alpha}\left(\lambda t_{1}^{\alpha}\right)\right|\left|\frac{x_{0}}{f\left(0, x_{0}\right)}\right| \\
\leq & \|h\|_{L^{1}} \int_{0}^{t_{1}}\left(t_{2}-s\right)^{\alpha-1} E_{\alpha, \alpha}\left(\lambda\left(t_{2}-s\right)^{\alpha}\right)-\left(t_{1}-s\right)^{\alpha-1} E_{\alpha, \alpha}\left(\lambda\left(t_{1}-s\right)^{\alpha}\right) d s \\
& +C_{1} \exp \left(2 \lambda^{\rho}\right) \int_{t_{1}}^{t_{2}} h(s) d s+\left|E_{\alpha}\left(\lambda t_{2}^{\alpha}\right)-E_{\alpha}\left(\lambda t_{1}^{\alpha}\right)\right|\left|\frac{x_{0}}{f\left(0, x_{0}\right)}\right| .
\end{aligned}
$$

In the other hand, we have

$$
\begin{aligned}
\int_{0}^{t_{1}}\left(t_{2}-s\right)^{\alpha-1} E_{\alpha, \alpha}\left(\lambda\left(t_{2}-s\right)^{\alpha}\right) d s & =-\int_{t_{2}}^{t_{2}-t_{1}} u^{\alpha-1} E_{\alpha, \alpha}\left(\lambda(u)^{\alpha}\right) d u=\int_{t_{2}-t_{1}}^{t_{2}} u^{\alpha-1} E_{\alpha, \alpha}\left(\lambda(u)^{\alpha}\right) d u \\
& =\int_{0}^{t_{2}} u^{\alpha-1} E_{\alpha, \alpha}\left(\lambda(u)^{\alpha}\right) d u-\int_{0}^{t_{2}-t_{1}} u^{\alpha-1} E_{\alpha, \alpha}\left(\lambda(u)^{\alpha}\right) d u
\end{aligned}
$$


According to the relation $(c)$ in lemma 2.2 , with $\beta=\alpha$, we obtain

$\int_{0}^{t_{1}}\left(t_{2}-s\right)^{\alpha-1} E_{\alpha, \alpha}\left(\lambda\left(t_{2}-s\right)^{\alpha}\right) d s=t_{2}^{\alpha} E_{\alpha, \alpha+1}\left(\lambda t_{2}^{\alpha}\right)-\left(t_{2}-t_{1}\right)^{\alpha} E_{\alpha, \alpha+1}\left(\lambda\left(t_{2}-t_{1}\right)^{\alpha}\right)$.

Similarly, we have:

$$
\int_{0}^{t_{1}}\left(t_{1}-s\right)^{\alpha-1} E_{\alpha, \alpha}\left(\lambda\left(t_{1}-s\right)^{\alpha}\right) d s=t_{1}^{\alpha} E_{\alpha, \alpha+1}\left(\lambda t_{1}^{\alpha}\right) .
$$

Finally,

$$
\begin{aligned}
\left|\left(\mathcal{B} x_{n}\right)\left(t_{2}\right)-\left(\mathcal{B} x_{n}\right)\left(t_{1}\right)\right| & \leq\left|E_{\alpha}\left(\lambda t_{2}^{\alpha}\right)-E_{\alpha}\left(\lambda t_{1}^{\alpha}\right)\right|\left|\frac{x_{0}}{f\left(0, x_{0}\right)}\right| \\
& +\|h\|_{L^{1}}\left[t_{2}^{\alpha} E_{\alpha, \alpha+1}\left(\lambda t_{2}^{\alpha}\right)-t_{1}^{\alpha} E_{\alpha, \alpha+1}\left(\lambda t_{1}^{\alpha}\right)+\left(t_{2}-t_{1}\right)^{\alpha} E_{\alpha, \alpha+1}\left(\lambda\left(t_{2}-t_{1}\right)^{\alpha}\right)\right] \\
& +C_{1} \exp \left(2 \lambda^{\rho}\right)\left|\psi\left(t_{2}\right)-\psi\left(t_{1}\right) \cdot\right|
\end{aligned}
$$

This implies that $\left|\left(\mathcal{B} x_{n}\right)\left(t_{2}\right)-\left(\mathcal{B} x_{n}\right)\left(t_{1}\right)\right| \rightarrow 0$ as $t_{2}-t_{1} \rightarrow 0$,

uniformly for all $\mathrm{n} \in \mathbb{N}$. This shows that the convergence $\mathcal{B} x_{n} \rightarrow B x$ is uniform and hence $\mathcal{B}$ is partially continuous on $E$.

Step $I V: \mathcal{B}$ is uniformly partially compact operator on $\mathrm{E}$.

Let $C$ be an arbitrary chain in $E$. We prove that $\mathcal{B}(C)$ is a uniformly bounded and equicontinuous set in E. First, we prove that $\mathcal{B}(C)$ is uniformly bounded. Let $y \in \mathcal{B}(C)$ be any element. Then, there is an element $x \in C$, such that $y=\mathcal{B} x$. By hypothesis $\left(B_{2}\right)$, for all $t \in J$,

$$
\begin{aligned}
|y(t)| & \leq\left|\int_{0}^{t}(t-s)^{\alpha-1} E_{\alpha, \alpha}\left(\lambda(t-s)^{\alpha}\right) g(s, x(s)) d s\right|+\left|\frac{x_{0}}{f\left(0, x_{0}\right)} E_{\alpha}\left(\lambda t^{\alpha}\right)\right| \\
& \leq C_{1} \exp \left(2 \lambda^{\rho}\right) \int_{0}^{1} h(s) d s+\left|\frac{x_{0}}{f\left(0, x_{0}\right)}\right| C_{1} \exp \left(2 \lambda^{\rho}\right) \\
& \leq C_{1} \exp \left(2 \lambda^{\rho}\right)\left(\|h\|_{L^{1}}+\left|\frac{x_{0}}{f\left(0, x_{0}\right)}\right|\right)=M,
\end{aligned}
$$

Thanks to the relation (d) in lemma 2.2 .

Taking supremum over $t$, we obtain $\|y\|=\|B x\| \leq M$ for all $y \in \mathcal{B}(C)$. Hence, $\mathcal{B}(C)$ is a uniformly bounded subset of E. Moreover, $\|\mathcal{B}(C)\| \leq M$ for all chains $\mathrm{C}$ in $\mathrm{E}$. Hence, $\mathcal{B}$ is a uniformly partially bounded operator on $\mathrm{E}$. 
Next, we will prove that $\mathcal{B}(C)$ is an equicontinuous set in $E$. Let $t_{1}, t_{2} \in J$ with $t_{1}<t_{2}$ and $\psi(t)=\int_{0}^{t} h(s) d s$. Then, for any $y \in \mathcal{B}(C)$, one has

$$
\begin{aligned}
\left|y\left(t_{2}\right)-y\left(t_{1}\right)\right| \leq & \left|\int_{0}^{t_{2}}\left(t_{2}-s\right)^{\alpha-1} E_{\alpha, \alpha}\left(\lambda\left(t_{2}-s\right)^{\alpha}\right) g(s, x(s)) d s-\int_{0}^{t_{1}}\left(t_{1}-s\right)^{\alpha-1} E_{\alpha, \alpha}\left(\lambda\left(t_{1}-s\right)^{\alpha}\right) g(s, x(s)) d s\right| \\
& +\left|E_{\alpha}\left(\lambda t_{2}^{\alpha}\right)-E_{\alpha}\left(\lambda t_{1}^{\alpha}\right)\right| \frac{x_{0}}{f\left(0, x_{0}\right)} \mid \\
\leq & \left|\int_{0}^{t_{1}}\left[\left(t_{2}-s\right)^{\alpha-1} E_{\alpha, \alpha}\left(\lambda\left(t_{2}-s\right)^{\alpha}\right)-\left(t_{1}-s\right)^{\alpha-1} E_{\alpha, \alpha}\left(\lambda\left(t_{1}-s\right)^{\alpha}\right)\right] g(s, x(s)) d s\right| \\
& +\left|\int_{t_{1}}^{t_{2}}\left(t_{2}-s\right)^{\alpha-1} E_{\alpha, \alpha}\left(\lambda\left(t_{2}-s\right)^{\alpha}\right) g(s, x(s)) d s\right|+\left|E_{\alpha}\left(\lambda t_{2}^{\alpha}\right)-E_{\alpha}\left(\lambda t_{1}^{\alpha}\right)\right|\left|\frac{x_{0}}{f\left(0, x_{0}\right)}\right| \\
\leq & \left|\int_{0}^{t_{1}}\right|\left(t_{2}-s\right)^{\alpha-1} E_{\alpha, \alpha}\left(\lambda\left(t_{2}-s\right)^{\alpha}\right)-\left(t_{1}-s\right)^{\alpha-1} E_{\alpha, \alpha}\left(\lambda\left(t_{1}-s\right)^{\alpha}\right)|| g(s, x(s))|d s| \\
& +\left|\int_{t_{1}}^{t_{2}}\left(t_{2}-s\right)^{\alpha-1} E_{\alpha, \alpha}\left(\lambda\left(t_{2}-s\right)^{\alpha}\right)\right| g(s, x(s))|d s|+\left|E_{\alpha}\left(\lambda t_{2}^{\alpha}\right)-E_{\alpha}\left(\lambda t_{1}^{\alpha}\right)\right|\left|\frac{x_{0}}{f\left(0, x_{0}\right)}\right| \\
\leq & \|h\|_{L^{1}} \int_{0}^{t_{1}}\left(t_{2}-s\right)^{\alpha-1} E_{\alpha, \alpha}\left(\lambda\left(t_{2}-s\right)^{\alpha}\right)-\left(t_{1}-s\right)^{\alpha-1} E_{\alpha, \alpha}\left(\lambda\left(t_{1}-s\right)^{\alpha}\right) d s \\
& +C_{1} \exp \left(2 \lambda^{\rho}\right) \int_{t_{1}}^{t_{2}} h(s) d s+\left|E_{\alpha}\left(\lambda t_{2}^{\alpha}\right)-E_{\alpha}\left(\lambda t_{1}^{\alpha}\right)\right|\left|\frac{x_{0}}{f\left(0, x_{0}\right)}\right| .
\end{aligned}
$$

Finally, according to the relation (3.6) and (3.7), we have

$$
\begin{aligned}
\left|y\left(t_{2}\right)-y\left(t_{1}\right)\right| & \leq\left|E_{\alpha}\left(\lambda t_{2}^{\alpha}\right)-E_{\alpha}\left(\lambda t_{1}^{\alpha}\right)\right|\left|\frac{x_{0}}{f\left(0, x_{0}\right)}\right| \\
& +\|h\|_{L^{1}}\left[t_{2}^{\alpha} E_{\alpha, \alpha+1}\left(\lambda t_{2}^{\alpha}\right)-t_{1}^{\alpha} E_{\alpha, \alpha+1}\left(\lambda t_{1}^{\alpha}\right)+\left(t_{2}-t_{1}\right)^{\alpha} E_{\alpha, \alpha+1}\left(\lambda\left(t_{2}-t_{1}\right)^{\alpha}\right)\right] \\
& +C_{1} \exp \left(2 \lambda^{\rho}\right)\left|\psi\left(t_{2}\right)-\psi\left(t_{1}\right)\right|
\end{aligned}
$$

This implies that $\left|y\left(t_{2}\right)-y\left(t_{1}\right)\right| \rightarrow 0$ as $t_{2}-t_{1} \rightarrow 0$,

uniformly for all $y \in \mathcal{B}(C)$. Hence $\mathcal{B}(C)$ is an equicontinuous subset of $E$. $\mathcal{B}(C)$ is a uniformly bounded and equicontinuous set of functions in $\mathrm{E}$, so it is compact. Consequently, $\mathcal{B}$ is a uniformly partially compact operator on $E$ into itself.

Step V: u satisfies the operator inequality $u \leq \mathcal{A} u \mathcal{B} u$.

By hypothesis $\left(B_{4}\right)$, the FQDE (1),(2) has a lower solution $u$ defined on $J$. Then, we have and

$$
D^{\alpha}\left(\frac{u(t)}{f(t, u(t)}\right)-\lambda\left(\frac{u(t)}{f(t, u(t)}\right) \leq g(t, u(t)), \text { for all } t \in J,
$$

$u(0) \leq x_{0}$.

Multiplying the above inequality by the integrating factor $E_{\alpha, \alpha}$, we obtain for all 
$t \in J$,

$$
\int_{0}^{t}(t-s)^{\alpha-1} E_{\alpha, \alpha}\left(\lambda(t-s)^{\alpha}\right)\left(D^{\alpha}\left(\frac{u(t)}{f(t, u(t)}\right)-\lambda\left(\frac{u(t)}{f(t, u(t)}\right)\right) d s \leq \int_{0}^{t}(t-s)^{\alpha-1} E_{\alpha, \alpha}\left(\lambda(t-s)^{\alpha}\right) g(s, u(s)) d s
$$

Using the relation (1) in lemma 2.1, with $n=1, U=1$ and $K=-\lambda$ one has

$$
\int_{0}^{t}(t-s)^{\alpha-1} E_{\alpha, \alpha}\left(\lambda(t-s)^{\alpha}\right) D^{\alpha} u(s) d s=u(t)-E_{\alpha}\left(\lambda t^{\alpha}\right) u(0)+\lambda \int_{0}^{t}(t-s)^{\alpha-1} E_{\alpha, \alpha}\left(\lambda(t-s)^{\alpha}\right) u(s) d s .
$$

Then we obtain:

$$
\frac{u(t)}{f(t, u(t))} \leq E_{\alpha}\left(\lambda t^{\alpha}\right) \frac{u(0)}{f(0, u(0))}+\int_{0}^{t}(t-s)^{\alpha-1} E_{\alpha, \alpha}\left(\lambda(t-s)^{\alpha}\right) g(s, u(s)) d s,
$$

for all $t \in J$. From the definitions of the operators $\mathcal{A}$ and $\mathcal{B}$, it follows that $u(t) \leq(\mathcal{A} u)(t)(\mathcal{B} u)(t)$, for all $t \in J$. Hence $u \leq \mathcal{A} u \mathcal{B} u$.

Step VI: The $\mathcal{D}$-function $\phi$ satisfies the growth condition $M \phi_{A}(r), r>0$.

The $\mathcal{D}$-function $\phi$ of the operator $\mathcal{A}$ satisfies the inequality given in hypothesis (c) of theorem 2.1. From the estimate (3.8), it follows that

$$
M \phi_{A}(r)=\left(C_{1} \exp \left(2|\lambda|^{\rho}\right)\left(\|h\|_{L^{1}}+\left|\frac{x_{0}}{f\left(0, x_{0}\right)}\right|\right)\right) \phi(r)<r, \text { for all } r>0 .
$$

Thus, $\mathcal{A}$ and $\mathcal{B}$ satisfy all the conditions of theorem 2.13 and we apply it to conclude that the operator equation $\mathcal{A} x \mathcal{B} x=x$ has a positive solution. Consequently, the integral equation (3.5) and the FQDE has a positive solution $x^{*}$ defined on $J$. Furthermore, the sequence $\left\{x_{n}\right\}_{n=1}^{\infty}$ of successive approximations defined by (3.4) converges monotonically to $x^{*}$. This completes the proof.

\section{REFERENCES}

[1] Bapurao C. Dhage, V. Lakshmikantham, Basic results on hybrid differential equations. Nonlinear Anal. Hybrid Syst.4, 414-424(2010)

[2] Bapurao C. Dhage, V. Lakshmikantham, Quadratic perturbations of periodic boundary value problems of second order ordinary differential equations. Differ. Equ. Appl.2, 465-486 (2010)

[3] Bapurao C. Dhage and Shyam B. Dhage, Approximating positive solutions of nonlinear first order ordinary quadratic differential equations: Applied and Interdisciplinary Mathematics, Cogent Mathematics, 2, 1023671(2015)

[4] Rudolf. Gorenflo, Anatoly A. Kilbas, Francesco Mainardi, Sergei V. Rogosin, Mittag-Leffler

Functions, Related Topics and Applications: Springer-Verlag Berlin Heidelberg, 2014

[5] K. Hilal,A. Kajouni, Boundary value problems for hybrid differential equations. Mathematical Theory and Modeling. 2224-5804 (2015)

[6] K. Hilal, A. Kajouni, Boundary value problems for hybrid differential equations with fractional order. Advances in Difference Equations. 183,2015 DOI 10.1186/s13662-015-0530-7.

[7] Heping Jiang, Existence results for fractional order functional differential equations with impulse, Computers and Mathematics with Applications 6434773483 (2012)

[8] A.A. Kilbas, H.M. Srivastava, J.J. Trujillo, Theory and Applications of Fractional Differential Equations, in North-Holland Mathematics Studies, vol. 204, Elsevier, Amsterdam, 2006. 
[9] Nickolai Kosmatov, Integral equations and initial value problems for nonlinear differential equations of fractional order, Nonlinear Anal. TMA (70) 25212529 (2009).

[10] V. Lakshmikantham, Theory of fractional functional differential equations, Nonlinear Anal. (2007), doi:10.1016/j.na.2007.09.025

[11] V. Lakshmikantham, A.S. Vatsala, Basic theory of fractional differential equations, Nonlinear Anal. (2007), doi:10.1016/j.na.2007.08.042

[12] Xiao-Li Ding1, Yao-Lin Jiang, waveform relaxation method for fractional differentialalgebraic equations, Fractional calculus and applied analysis, vol 17, 3 (2014)

[13] K.S. Miller, B. Ross, An Introduction to the Fractional Calculus and Fractional Differential Equations, Wiley, New York, 1993

[14] I. Podlubny, Fractional Differential Equations, Academic Press, New York, 1993.

[15] Yong Zhou, Feng Jiao, Jing Li, Existence and uniqueness for fractional neutral differential equations with infinite delay, Nonlinear Anal. TMA 7132493256 (2009)

[16] Yong Zhou, Basic theory of fractional differential equations, Xiangtan University, China, 2014.

(K. Hilal) Sultan Moulay Slimane university, Laboratoire de Mathématiques Appliquées \& Calcul Scientifique, BP 523, 23000, Beni Mellal, Morocco

E-mail address, K. Hilal: hilal.khalid@yahoo.fr

(Y. Allaoui) Sultan Moulay Slimane university, Laboratoire de Mathématiques ApPliquées \& Calcul Scientifique, BP 523, 23000, Beni Mellal, Morocco

E-mail address, Y. Allaoui: youssefbenlarbi1990@gmail.com

(K. Guida) Sultan Moulay Slimane university, Laboratoire de Mathématiques ApPliquées \& Calcul Scientifique, BP 523, 23000, Beni Mellal, Morocco

E-mail address, K. Guida: guida.karim@gmail.com 